\title{
E-monitoring and Tele-orthodontics of a Patients with Crossbite During Covid-19 Pandemic - A Case Report
}

\author{
Krishna Arora* \\ Associate Professor, Department of Orthodontics, Manipal University College, \\ Malaysia \\ *Corresponding Author: Krishna Arora, Associate Professor, Department of \\ Orthodontics, Manipal University College, Malaysia.
}

Received: October 01, 2021

Published: October 27, 2021

(c) All rights are reserved by Krishna Arora.

\begin{abstract}
This article reports a treatment carried out on 20-years-old female Chinese patient with aesthetic and functional complaints. She presented with Angle's Class-I malocclusion, crossbite and moderate crowding in maxillary arch, in addition to a slightly incompetent lip. Crossbite and crowding were corrected by dental expansion using self-ligating brackets, which not only contributed to alignment and levelling of teeth but also ensuring less plaque build-up and inflammation, considering poor oral hygiene of the patient. In addition, this appliance also helps during pandemic ensuring less frequent visit to the dental clinic and better overall maintenance. Thanks to the current generation sophisticated mobile and internet connectivity, tele-orthodontics ensured the wellbeing and progress of treatment using e-monitoring. Upon treatment completion, patient's self-esteem had significantly improved, good facial proportions and fuller lips were observed. Patients should be carefully monitored during adolescence to avoid such complex treatments and electronic platforms like video call, pictures sharing in proper format help tracking the progress and avoid any future complication amid Covid-19 crisis.
\end{abstract}

Keywords: Crossbite; Self-ligating; Fixed Orthodontic Treatment; Tele-orthodontics

\section{Abbreviations}

TMJ: Temporomandibular Joint; COVID-19: Coronavirus Disease; e-mail: Electronic Mail; SLB: Self Ligating Brackets; Cu-Niti: Copper-Nickel Titanium; SS: Stainless Steel; Apps: Mobile Applications; MCO: Movement Control Order; SOP: Standard Operating Procedures; e-monitoring: Electronic/Remote Monitoring.

\section{Introduction}

Anterior crossbite refers to malocclusion resulting from lingual position of maxillary anterior teeth in relationship to the mandibular anterior teeth. The reported prevalence of anterior crossbite in the mixed dentition varies between 1.6 per cent and 7.9 per cent [1-4]. Most commonly found in Class-III patients with a rarer oc- currence among other classes. Crossbite can cause excessive attrition, poor masticatory efficiency, poor gingival health and TMJ problems [5]. Crowding can occur due to discrepancy between Arch width-tooth material/Arch length-tooth material. Treatment via fixed orthodontic appliances is indicated due to ease of use and availability [6].

Amid COVID-19 emergency, dental offices suspended all deferrable procedures to reduce the spread of the pathogen. As of now, dental emergencies are managed via remote triage (phone calls, email, or other online modalities). But how digital techniques can help us in the prosecution of our clinical activity during prolonged emergency lockdowns and in the future? Every patient was sup- 
posed to have their monthly check-ups, but an in-person visit with the orthodontist was not permitted during the COVID-19 lockdown, and only non-deferrable healthcare (urgent care) was allowed to be offered. According to the American Dental Association, the substitution of metal wires causing mucous ulcers is the only orthodontic procedure considered non-deferrable [7].

Tele-orthodontics is a broad term that encompasses remote provision of orthodontic care, advice, or treatment via information technology [8]. In the early to mid-2000s, promising results were achieved by orthodontists supervising general dental practitioners in real time to provide orthodontic services to patients with limited access to orthodontic care $[9,10]$. Simple remote monitoring of patients during the retention period has also been performed with patients sending pictures instead of travelling for in-office visits. The application of the "tele-orthodontics" concept however has been limited thus far. With the advent of tele-orthodontics, and more specifically remote monitoring, the scheduling of in-office visits can be personalized per patient, creating a supposedly more efficient workflow. This not only maximizes profitability by reducing chair time, but also improves patient convenience. Other perceived advantages include earlier diagnoses, closer management through remote monitoring, savings in time and transportation costs and increased convenience for patients [11]. Remote patient monitoring may also reduce overall treatment time via early interception of problems such as non-tracking aligners, debonded brackets or broken appliances, allowing for such problems to be addressed promptly. Our tele-orthodontics protocol included options such as video calls (Zoom Video Communications, Inc.) sharing of intraoral and extraoral photos and instant messaging. For fixed orthodontic therapy with brackets, the photos made it possible to check the progression of the treatment, but all the issues needing a direct intervention of the orthodontist were not possible and required troubleshooting and planning.

This case report presents a 20-year-old female reported to the department of Orthodontics, Faculty of Dentistry, Melaka-Manipal Medical college (now Manipal university college Malaysia) for the following problem: "I am shy of smiling and feel uneasy and I want to have my teeth fixed because they are not aligned, and which makes it difficult to chew".
Diagnosis

Patient was diagnosed as Angle's Class-I malocclusion with crossbite in the canine regions anteriorly and premolar region in the posterior right quadrant, narrow maxillary arch with crowding. Detailed clinical examination revealed a normal Class-I molar relationship, 12,13,14,15 22 and 25 were in crossbite in relation with its lower counterpart (Figure 2). Upper dental midline was shifted to left by $2.5 \mathrm{~mm}$, reduced overjet and almost reverse overbite. The crowding was found to be $5.5 \mathrm{~mm}$ in maxillary arch. Extraoral pictures reveals patient's lips looks incompetent and shy of smiling (Figure 1).

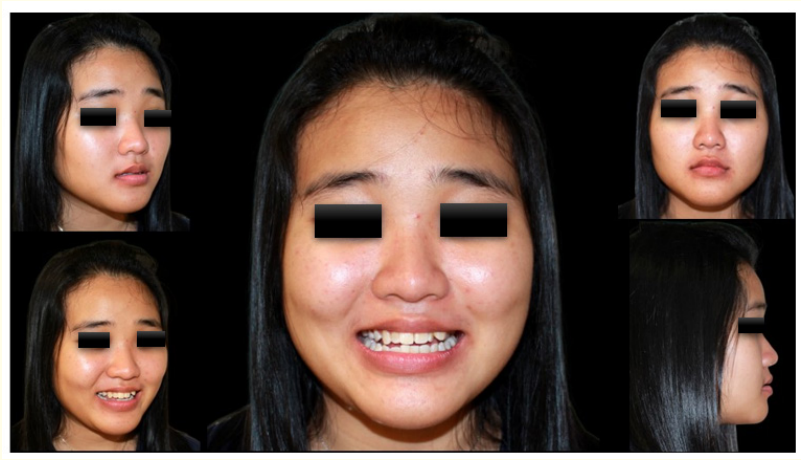

Figure 1

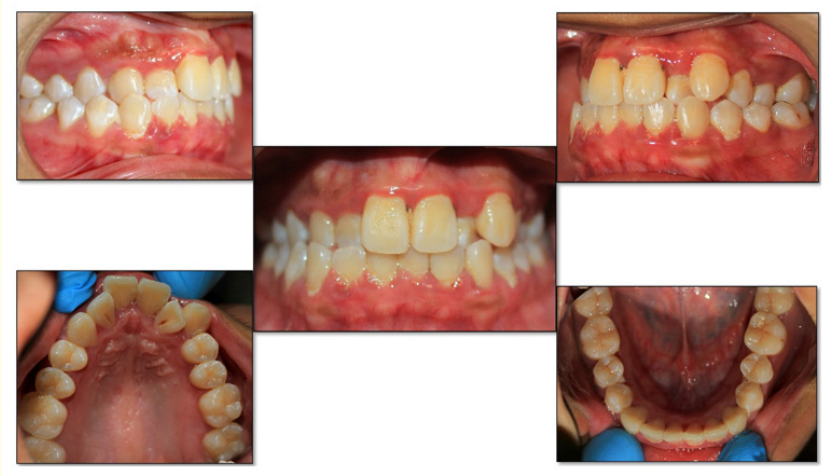

Figure 2 


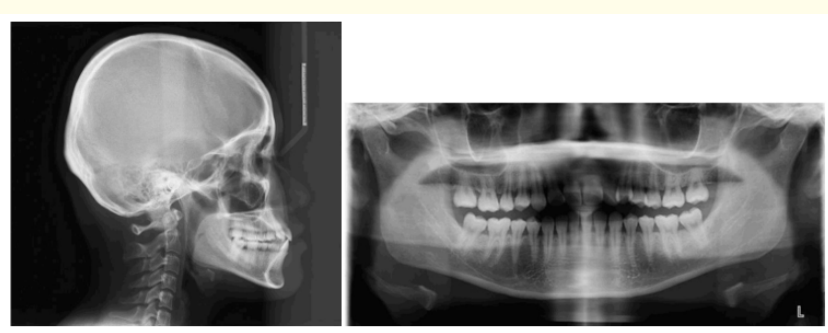

Figure 3

\section{Treatment objectives}

To correct the crossbites, relieve the crowding and align the anterior segment to achieve Class I incisor and canine relationship with complete interdigitation of entire dentition.

\section{Treatment plan}

After performing cephalometric analysis and study model analysis it was decided to treat the patient with non-extraction protocol utilizing the arch arch-expansion advantage of self-ligating brackets $[12,13]$.

Treatment plan aimed at relieving the crossbite by means of a fixed orthodontic appliance, as an obvious choice providing a little labial flaring of anterior teeth in ideal occlusion and expansion of upper arch. The fixed orthodontic appliance was bonded on the upper arch only to allow proper expansion and to relieve crowding followed by the lower arch later as the correction of upper arch progresses using self-ligating (Damon Q) brackets. Levelling and alignment were achieved with Damon Cu-Niti arch wires, starting with round 0.014 wire for 4 weeks. Open coil springs was placed between 21-23 to create space for 22 using round 0.016 ss for 6 weeks. 22 was then ligated into the slot using round 0.14 overlay wire and 0.016 ss wire continued for 6 weeks along with open coil spring. $0.016 \times 0.025$ rectangular $\mathrm{Cu}-\mathrm{Niti}$ was given after 8 weeks to continue the arch expansion for 6 weeks. Posterior bite block of glass ionomer cement has been given for $4+4$ weeks to provide clearance and expansion of maxillary teeth. $0.018 \times 0.025 \mathrm{Cu}-\mathrm{Niti}$ given for another 4 weeks also lower arch has been bonded and $0.014 \mathrm{Cu}$-Niti given for 4 weeks. Next visit ss rectangular wire has been placed in upper arch and lower arch changed to $0.016 \times 0.025$
$\mathrm{Cu}-\mathrm{Niti}$ for 4 weeks. Next appointment both the arches were ligated with ss wires and cross elastic given to correct the midlines. As we were approaching the end of treatment Covid-19 pandemic hits hard and the clinics need to shut down following MCO of the country and in-person visit with the orthodontist was not permitted during the lockdown, and only non-deferrable healthcare (urgent care) was allowed to be offered according to the American Dental Association. So, we decided to review all our cases through electronic media including picture sharing through messaging applications and e-mail. Once the MCO was lifted patient was recalled for review and immediately changed the wires to final settling wire for finishing and detailing and settling elastic has been started. But unfortunately, due to second MCO in place the clinic needs to be closed again. We continued guiding and review of patient through electronic media this time including zoom calls, for those who are having tough time following the instructions (tele-orthodontics).

Appliance was debonded immediately following the lifting of lockdown and bonded retainers were given. Tele-orthodontics not only helped remote monitoring of the patient but also comes handy in avoiding any mishap or nuisance. Though the total time of the treatment took almost two years but the number of visits by the patient to clinic were just ten, considering the unavoidable circumstances of pandemic which prolonged the time duration. Currently we are facing the third MCO and hence patient has been scheduled for post retention check-up after the upliftment of MCO.

\section{Results}

At treatment completion, patient's self-esteem had significantly improved. Good facial proportions were observed in frontal and oblique view, (Figure 4) with patient's profile improved due to an increase in lip volume. The incompetency of the lip also has been addressed and lips look fuller and competent. Normal molar and canine relationships were obtained as a key to occlusion. Crossbite and crowding were corrected by dental expansion of the maxillary arch (Figure 5). Normal overjet and overbite were obtained, and normal midline was restored at the end of the treatment.

\section{Discussion}

Nowadays patients are more aware about aesthetic or appearance in contrast to the ideal orthodontic norms of occlusion. Though crossbites not just affect the appearance but seriously af- 


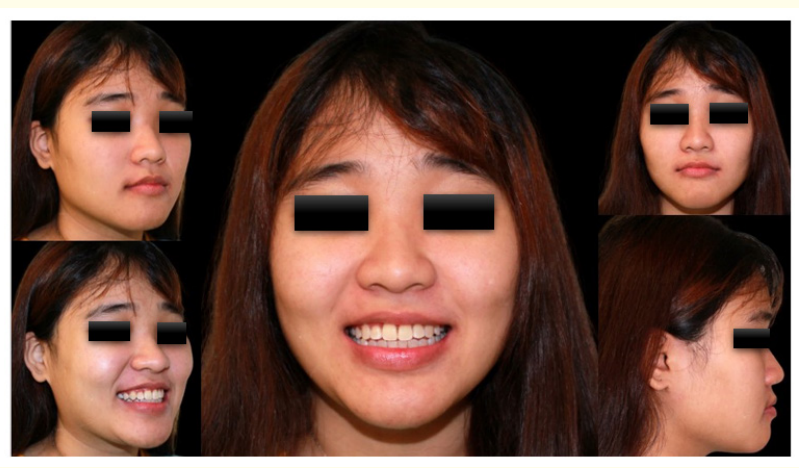

Figure 4

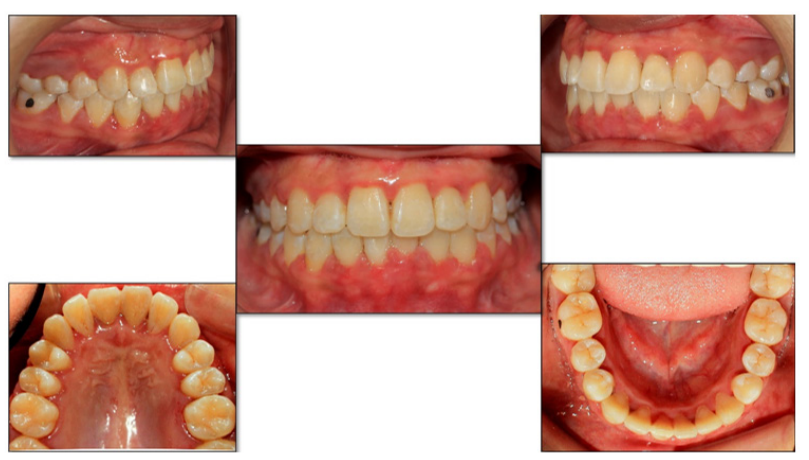

Figure 5
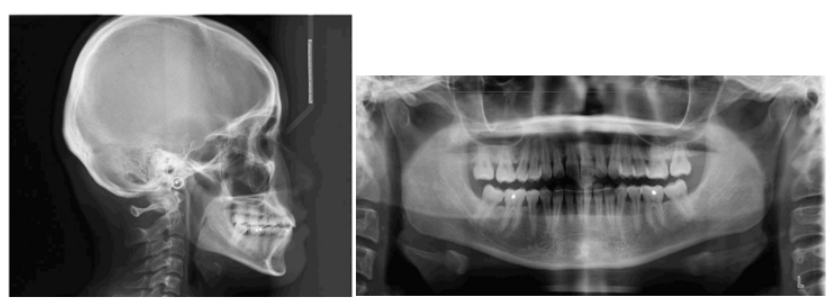

Figure 6

fect the occlusion. Some ethnicities due to certain environmental or biological factors are predisposed to develop certain dental anom- alies [14]. Comprehensive orthodontic treatment needs tedious and time-consuming efforts to achieve the perfect ideal occlusion whereas minor tooth movement allows a direct approach to address the patient's primary concern with minimal adjunctive therapy. Anterior crossbite must be treated in primary or mixed dentition, if not it may obstruct the normal physiological development of the jaws and leaving them till the eruption of permanent dentition can lead to very limited options of treatment provision. Correction of moderate crowding or arch length-tooth material discrepancy/ arch width-tooth material discrepancy can be managed by dental expansion or proclination of incisors and can contribute to obtain well aligned arches without extraction of any permanent tooth. Ideal results can be achieved by enforcing isolated movements to single tooth effortlessly. SLB helps achieving dental expansion and relief from crowding efficiently compared to traditional brackets. It's also ideal for patients who come from nearby towns, and provinces and wants to reduce the number of visits and frequent travelling. SLB also a choice of appliance in patients with poor oral hygiene like this patient education and compliance plays an important role towards better and more stable outcomes. Patient should be continuously motivated and followed up to avoid relapse or minor complication which could jeopardize the quality of treatment provided. Covid-19 pandemic possesses a possible threat of failure to achieve ideal results of orthodontic treatment especially those are based on patient's compliance due to frequent lockdowns and changes in protocols, hence tele-orthodontics becomes so important and helps a clinician to keep a track of progress of treatment remotely and will be a beneficial and an effective way of communication or record keeping tool in coming future.

\section{Conclusion}

Patients should be monitored carefully and frequently during adolescence to avoid later complex treatments. If patient was referred to a specialist early, or reported to a clinic early, full fixed appliance could have been avoided. As our patient was adult on arrival, treatment was planned and executed with limited alternatives but set goals were accomplished. Patient monitoring now plays more important role as we all are dealing with a pandemic with lots of restriction and SOPs to follow and in future, we are not sure to not to observe such kind of pandemics, so early adoption of e-monitoring will not only help a clinician to keep a track of progress of treatment but also for patient it reduces number of 
visit to clinic. Simple instruction of changing the elastics, increasing of reducing the force level of elastics, patient ready to be recalled for review or not etc. are such few examples of e-monitoring or tele-orthodontics. Further collaboration with the application developers is required to bring out the best possible dental monitoring system solution.

\section{Bibliography}

1. Keski-Nisula K., et al. "Occurrence of malocclusion and need of orthodontic treatment in early mixed dentition". American Journal of Orthodontics and Dentofacial Orthopedics 124 (2003): 631-638.

2. Dimberg L., et al. "Malocclusions in children at 3 and 7 years of age: a longitudinal study". European Journal of Orthodontics 35 (2013): 131-137.

3. Lux CJ., et al. "Occlusal status and prevalence of occlusal malocclusion traits among 9-year-old schoolchildren". European Journal of Orthodontics 31 (2009): 294-299.

4. Schopf P. "Indication for and frequency of early orthodontic therapy or interceptive measures". Journal of Orofacial Orthopedics 64 (2003): 186-200.

5. Chintawongvanich J and Thongudomporn U. "Arch Dimension and Tooth Size in Class I Malocclusion Patients with Anterior Crossbite". Journal of the Dental Association of Thailand 63.1 (2013): 31-38.

6. Krishna Arora. "Minimalistic approach for the correction of anterior crossbite- a case report". European Journal of Biomedical and Pharmaceutical Sciences 5.2 (2018): 1147-1150.

7. S Saccomanno., et al. "Perspectives of tele-orthodontics in the COVID-19 emergency and as a future tool in daily practice". European Journal of Paediatric Dentistry 21.2 (2020): 157-162.

8. Ismaeel Hansa., et al. "Remote monitoring and "Tele-orthodontics": Concept, scope and applications". Seminars in Orthodontics 24.4 (2018): 470-481.

9. Berndt J., et al. "Using teledentistry to provide interceptive orthodontic services to disadvantaged children". American Journal of Orthodontics and Dentofacial Orthopedics 134.5 (2008): 700-706.
10. Stephens C., et al. "Orthodontic referrals via TeleDent Southwest”. Dental Clinics of North America 46.3 (2002): 507-520.

11. Dunbar AC., et al. "The influence of using digital diagnostic information on orthodontic treatment planning - a pilot study". Journal of Healthcare Engineering 5.4 (2014): 411-427.

12. Dr Anshu Singh., et al. "Effects of Different Ligation Systems on Arch Expansion, Incisor Inclination and Treatement Duration in Patients with Mild to Moderate Crowding Patients- A Prospective Study". Saudi Medical Journal 5.10 (2020): 316-318.

13. Naiara Carolina Jacob Lima., et al. "Comparison of Changes in Dental Arch Dimensions in Cases Treated with Conventional Appliances and Self-Ligating Damon System". The Open Dentistry Journal 12 (2018): 1137-1146.

14. Alam MK., et al. "Changes of lip morphology in relation to different skeletal index: in malaysian malay and malaysian chinese population". International Journal of Pharma and Bio Sciences 6.2 (2015): 770-778.

\section{Volume 5 Issue 11 November 2021 \\ (C) All rights are reserved by Krishna Arora.}

